\title{
Magnetohydrodynamic waves in a sheared potential coronal arcade
}

\author{
I. Arregui ${ }^{\star}$, R. Oliver, and J. L. Ballester
}

\begin{abstract}
Departament de Física, Universitat de les Illes Balears, 07122 Palma de Mallorca, Spain e-mail: Inigo.Arregui@wis.kuleuven.ac.be; [ramon.oliver;dfsjlbo]@uib.es
\end{abstract}

Received 30 March 2004 / Accepted 21 June 2004

\begin{abstract}
We study the effects of magnetic field shear $\left(B_{y} \neq 0\right)$ and longitudinal propagation of perturbations $\left(k_{y} \neq 0\right)$ on the linear and adiabatic magnetohydrodynamic (MHD) normal modes of oscillation of a potential coronal arcade. In a cold plasma, the inclusion of these two effects produces the linear coupling of discrete fast modes, characterised by a discrete spectrum of frequencies and a global velocity structure, and Alfvén continuum modes, characterised by a continuous spectrum of frequencies and with a velocity perturbation confined to given magnetic surfaces in such a way that modes with mixed properties arise (Arregui et al. 2004). The wave equations governing the velocity perturbations have been solved numerically and our results show that the couplings between fast and Alfvén modes are governed by some parity rules for the symmetry of the eigenfunctions of fast and Alfvén modes in the direction along the equilibrium magnetic field. The nature of the coupling between fast and Alfvén modes can be resonant or non-resonant depending on the location of the fast mode frequency within the different Alfvén continua. Also, an important result is that in this kind of configurations coupled modes could be difficult to observe since when both magnetic field shear and longitudinal propagation are present the spatial distribution of the velocity may not be confined to low heights in the solar corona.
\end{abstract}

Key words. magnetohydrodynamics (MHD) - methods: numerical - Sun: oscillations - Sun: magnetic fields

\section{Introduction}

Although first detected in the early 70s mainly in the radio band (see Aschwanden 1987, for a review), oscillations in solar coronal magnetic structures have been unambiguously observed due to the increased spatial and temporal resolution of imaging telescopes on board spacecrafts such as SOHO and TRACE. Oscillations have been detected in coronal loops (Nakariakov et al. 1999; Aschwanden et al. 1999; De Moortel et al. 2000; Robbrecht et al. 2001), coronal holes (Ofman et al. 2000), coronal plumes (DeForest \& Gurman 1998) and also in coronal arcades (see e.g. the "harmonica" event observed on April 15, 2001 by TRACE). Observations carried out with these instruments have allowed to report oscillatory phenomena in practically all wavelengths and have provided with a wealth of information about some of their properties such as the wavelengths, propagation speeds, amplitudes, damping times, etc. Regarding the nature of these oscillations, both propagating waves (Berghmans \& Clette 1999; De Moortel et al. 2002b,c) as well as standing oscillations (Wang et al. 2003) have been observed, pointing out the fact that the magnetically dominated solar corona harbours different dynamical and oscillatory phenomena.

^ Present address: Centre for Plasma Astrophysics, K.U. Leuven, Celestijnenlaan 200B, 3001 Heverlee, Belgium.
Most of these observations have been interpreted in terms of standing or propagating magnetohydrodynamic waves. Just like the first signatures for oscillatory motions observed in the radio band (Rosenberg 1970; Aschwanden 1987) stimulated the theoretical modelling of MHD waves in structured media (see Roberts et al. 1984, for example), the recent observational achievements have motivated an increasing effort from the theoretical point of view, leading to a refinement of the models that try to explain and even predict these observations. These facts have motivated coronal seismology, i.e. the probing of the physical properties of the corona by means of the study of the oscillations of its magnetic structures, to become an important field of research. Coronal seismology has already been applied by a number of authors for the estimation of different physical parameters in the solar corona. For example, Nakariakov et al. (1999) used this method for the estimation of the coronal dissipative coefficients. Nakariakov \& Ofman (2001) showed that the Alfvén speed and magnetic field strength in coronal loops can be estimated, using data from flare-generated loop oscillations. De Moortel et al. (2002a) have shown that the period, damping coefficient and decay exponent of loop oscillations can be determined by means of the wavelet analysis, although they have also stressed that a sufficient number of oscillations must be present in the analysed time series to obtain correct values of these parameters. By using novel data analysis techniques, Terradas (2002) quantified the properties 
of the oscillatory motions of coronal loops by means of twodimensional maps of the distribution of amplitudes inside the loop structures. His results indicate that the larger amplitudes of a propagating feature are located close to the edge of the loop. Also, a filamentary pattern inside the loop, probably associated with its fine structure, was found. Finally, Zaqarashvili (2003) suggested that time series spectroscopic observations at different heights above an active region at the solar limb may allow to determine the period and wavelength of global torsional oscillations and consequently the Alfvén speed in the corona. Indeed, from the analysis of early observations by Egan \& Schneeberger (1979) and assuming a constant value of the Alfvén speed along the loop, Zaqarashvili (2003) obtained a value of the Alfvén speed in the corona of $500 \mathrm{~km} \mathrm{~s}^{-1}$.

The aforementioned results are based on the detection of MHD waves in coronal structures together with the application of theoretical models to extract information on the physical parameters of interest. Nevertheless, for the development of an accurate coronal seismology the two activities, observations and theory, should mature to a sufficiently high degree so as to allow for a unique correlation between calculated and observed parameters. This paper aims to contribute to the theoretical side of coronal seismology by studying the MHD normal modes of oscillation of solar coronal arcades. Normal mode analysis provides with a physical basis for the understanding of the dynamics of the system. Previous theoretical works have already given some insights into the subject of the normal modes of oscillation of two-dimensional magnetic configurations embedded in a plasma. Oliver et al. (1993) studied analytically the modes of oscillation of a potential arcade for different density profiles and boundary conditions. This work was later extended by considering the MHD modes of oscillation of non-potential arcades in the absence of gravity (Oliver et al. 1996). Terradas et al. (1999) obtained numerical solutions for a fully magnetostatic arcade with gravity and concluded that a necessary, although not sufficient, requirement for fast mode oscillations to be confined in the corona is that the Alfvén speed must increase with height. On the other hand, the existence of singular solutions of the wave equations for particular magnetic field geometries and particular variations of the equilibrium plasma quantities, such as the Alfvén speed, has also been extensively studied not only in the solar, but also in the fusion research context (Goossens et al. 1985; Mond et al. 1990; Hansen \& Harrold 1994). In this respect, Mond et al. (1990) demonstrated that a singularity is present if the equilibrium magnetic field is straight and the Alfvén speed does not vary along field lines and also, although in this case it is not so apparent, when the magnetic field is not straight or the Alfvén speed varies along the equilibrium magnetic field. In this way, the slow and Alfvén continuous spectra have been determined for several two-dimensional equilibrium configurations (see Poedts \& Goossens 1987, 1991; Ruderman et al. 1997, for example). These works have provided with the possibility of investigating physical mechanisms for the deposition of energy via resonant absorption of waves in the context of solar coronal heating.

With the purpose of extending the normal mode analysis initiated by Oliver et al. (1993), Arregui et al. (2001) derived the linear MHD wave equations for a general, two-dimensional, sheared, force-free magnetic field configuration and developed a numerical code to solve the resulting system of coupled partial differential equations. Later, Arregui et al. (2003) modified the code in order to properly compute the two-dimensional spatial structure of modes with regular singularities and studied in detail the coupling of fast and Alfvén modes in a simple straight, uniform and bounded magnetic field configuration with density stratification in the vertical direction. Their results already pointed out that, from the observational point of view, the identification of modes present even in such an oversimplified magnetic configuration is not an easy task. One may then wonder what problems are to be faced in the analysis of the oscillatory modes of real coronal objects, in which structuring and curvature play an important role. Very recently, a more realistic sheared force-free curved arcade configuration was considered by Arregui et al. (2004). These authors discussed the resonant and non-resonant coupling of fast and Alfvén modes and showed that the coupling between fast and Alfvén modes is governed by some parity rules of the eigenfunctions with respect to the direction of the equilibrium magnetic field. The longitudinal magnetic field component produces the coupling of modes with opposite parity, whereas the presence of longitudinal wave propagation allows only for the coupling of fast and Alfvén modes with the same parity. When both effects are present, mode coupling can take place between modes of equal or opposite parity, enormously complicating the resulting spectrum and spatial structure of the perturbed velocity components. Our aim in this paper is to study the properties of linear MHD waves in a sheared coronal potential arcade in which constitutes an extension to the work by Oliver et al. (1993) to the cases in which both magnetic shear and/or longitudinal propagation are taken into account. Finally, this study can allow for a comparison between the normal modes of oscillation of the sheared potential equilibrium considered here and the linear force-free configuration studied by Arregui et al. (2004).

The paper is organised as follows. In Sect. 2 the basic equations describing the equilibrium configuration (Sect. 2.1) and the linear MHD wave equations for the normal modes of oscillation of this equilibrium (Sect. 2.2) are shown. Also, the numerical method and the boundary conditions used to solve these equations are described (Sect. 2.3). The results of the paper are given in Sect. 3. First, the effect of magnetic field shear is studied (Sect. 3.1) and then, in Sect. 3.2, the combined effect of shear and longitudinal propagation on the oscillatory properties of fast and Alfvén modes is described. Finally, in Sect. 4 conclusions are drawn.

\section{Basic equations and numerical method}

\subsection{Equilibrium configuration}

We consider that our coronal magnetic arcade configuration can be modelled by means of a two-dimensional, $y$-invariant, force-free magnetic field satisfying

$(\nabla \times B) \times \boldsymbol{B}=\mathbf{0}$. 
From the solenoidal condition, $\nabla \cdot \boldsymbol{B}=0$, together with the assumed $y$-invariance of the equilibrium, this magnetic field can be expressed in the following form

$\boldsymbol{B}=\nabla A(x, z) \times \hat{\boldsymbol{e}}_{y}+B_{y} \hat{\boldsymbol{e}}_{y}=B_{p} \hat{\boldsymbol{e}}_{p}+B_{y} \hat{\boldsymbol{e}}_{y}$,

with a component in the poloidal direction, locally parallel to the poloidal magnetic field and contained in the $x z$-plane, and a component in the longitudinal $\left(\hat{\boldsymbol{e}}_{y}\right)$ direction. The poloidal component of the magnetic field is determined by the flux function, $A(x, z)$, which is the $y$-component of the potential vector $\boldsymbol{A}$. In Cartesian coordinates the magnetic field can be written as

$\boldsymbol{B}=\left(-\frac{\partial A}{\partial z}, B_{y}, \frac{\partial A}{\partial x}\right)$,

where

$B_{y}=\frac{\partial A_{x}}{\partial z}-\frac{\partial A_{z}}{\partial x}$.

The force-free condition (Eq. (1)) dictates that $B_{y}(x, z)$ is a strict function of $A(x, z)$ and that the two are related by the Grad-Shafranov equation,

$\nabla^{2} A(x, z)+B_{y}(A) \frac{\mathrm{d} B_{y}(A)}{\mathrm{d} A}=0$,

which determines the flux function, $A(x, z)$, once the function $B_{y}(A)$ has been prescribed. Analytical solutions to Eq. (5) are known for particular forms of $B_{y}(A)$, such as $B_{y}(A)=$ constant. In this case, from Eq. (5) the flux function must satisfy

$\nabla^{2} A(x, z)=0$

and we have a so-called potential magnetic field. A solution to Eq. (6) can be obtained by separation of variables under the conditions that it does not diverge at infinity and that the resulting vertical component of the magnetic field vanishes at $x=0$ (i.e. a symmetry condition). Then, the full solution for $A(x, z)$ is obtained by summing over all possible solutions,

$A(x, z)=\sum_{n} A_{n} \cos k x \mathrm{e}^{-k z}$,

with $k=n \pi / 2 L$. If we assume that only one Fourier component is taken, the potential solution is simply given by

$A(x, z)=\frac{B_{0}}{k} \cos k x \mathrm{e}^{-k z}$,

where $B_{0}$ is the magnetic field strength at $x=0, z=0$ and $k^{-1}=2 L / \pi$ the magnetic scale height. Now, the magnetic field components can be obtained from Eqs. (3) and (4), which result in

$B_{x}=B_{0} \cos k x \mathrm{e}^{-k z}$,

$B_{y}=c B_{0}$,

$B_{z}=-B_{0} \sin k x \mathrm{e}^{-k z}$,

with $c$ being a constant.

The value of the longitudinal magnetic field component in Eq. (10) can be either zero or any other constant. If $B_{y}=0$, then the potential field is purely contained in the $x z$-plane. The modes of oscillation of such a poloidal potential coronal arcade were studied by Oliver et al. (1993) in the cold plasma limit for different values of the density profile and boundary conditions. This equilibrium was also used by Smith et al. (1997) and Tirry $\&$ Poedts (1998). In general, however, $B_{y}$ is a non-zero constant and field lines are sheared with a shear angle, $\gamma$, given by

$\gamma=\arctan \left(\frac{B_{y}}{B_{x}}\right)=\arctan \left(\frac{c}{\cos k x \mathrm{e}^{-k z}}\right)$.

Equation (12) indicates that the shear only depends on the flux function, given by Eq. (8), and so it is uniform on each magnetic surface. This expression also tells us that field lines on higher magnetic surfaces (i.e. for smaller values of the flux function) have a larger shear than those belonging to low-lying magnetic surfaces. The shear angle at the origin, $\gamma_{0}=\gamma(x=$ $0, z=0)$, is given by $\tan \left(\gamma_{0}\right)=c$ and then the vertical variation of the shear at $x=0$ is given by $\tan (\gamma)=\tan \left(\gamma_{0}\right) \mathrm{e}^{k z}$, so the shear increases rapidly with height.

The abovementioned properties of our magnetic equilibrium are displayed in Fig. 1. Figure 1a shows magnetic field lines belonging to three different magnetic surfaces, i.e. with three different $x$-coordinates of the footpoint position, $x_{0}$. By virtue of Eq. (12) these field lines are differently sheared when emerging upwards from the photosphere, even if in this case the shear angle at the origin $\gamma_{0}$ is the same for field lines on all three magnetic surfaces. On the other hand, in Fig. 1b magnetic field lines belonging to the same magnetic surface have been depicted for three different values of the shear angle at the origin, $\gamma_{0}$. In both plots, the projection of some field lines in the $x y$ - (photosphere), $x z$ - and $y z$-planes are also shown. Also important is the fact that contrary to what happens in the forcefree equilibrium considered by Arregui et al. (2004), in this magnetic equilibrium the shear changes from one magnetic surface to another. In addition, in the present configuration both the horizontal and vertical magnetic scale heights are equal while in a linear force-free magnetic configuration the constant shear is indeed given by the ratio of these two quantities.

The description of our equilibrium configuration is completed with an expression for the equilibrium Alfvén speed. Since we are neglecting the gas pressure and gravity terms in the force-balance equation, the density does not appear in Eq. (1) and we can impose arbitrarily the equilibrium density or Alfvén speed. Here, we have considered that the squared Alfvén speed has a vertical dependence of the form

$v_{\mathrm{A}}^{2}(z)=v_{\mathrm{A} 0}^{2} \mathrm{e}^{-(2-\delta) k z}$,

where $\delta$ is a parameter that determines the rate of change of the Alfvén speed with height from the value $v_{\mathrm{A} 0}$ at $z=0$. In a potential arcade with no longitudinal magnetic field component $\left(B_{y}=0\right)$, Eq. (13) gives the variation of the Alfvén speed with height when an exponentially decreasing equilibrium density is considered. The parameter $\delta$ then represents the ratio of the magnetic field scale height to the density scale height. In general, the longitudinal magnetic field component is not zero $\left(B_{y} \neq 0\right)$ and the Alfvén speed profile given by Eq. (13) is imposed rather than obtained. As a consequence, the equilibrium density still has a pure dependence in the vertical direction, $\rho=\rho(z)$, although this is not given by the simple relation representing an exponentially decaying function. The reason for 


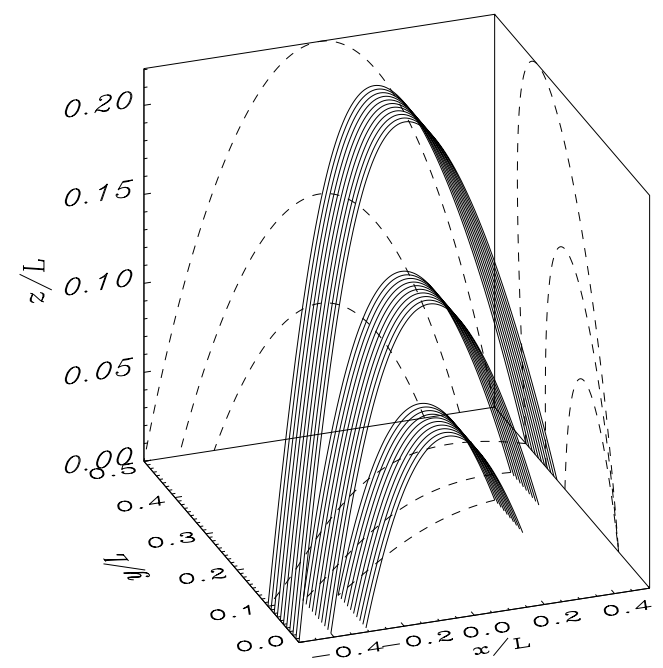

(a)

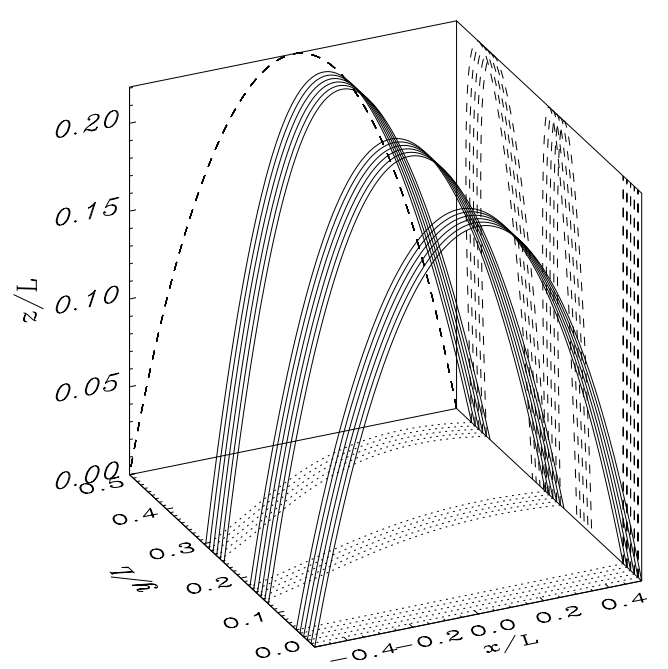

(b)

Fig. 1. Three-dimensional view of some magnetic field lines in a sheared potential coronal arcade. a) Several field lines corresponding to three different magnetic surfaces (field lines with three different footpoint positions; $x_{0} / L=0.3, x_{0} / L=0.4$ and $x_{0} / L=0.5$ ) with the same shear angle at the origin $\left(\gamma_{0}=30^{\circ}\right)$ are differently sheared as they emerge upwards. The projection of one of these field lines onto $x y$ - (photosphere), $x z$ - and $y z$-planes is also shown. b) The magnetic field lines corresponding to the same magnetic surface (same footpoint position $x_{0} / L=0.5$ ) are plotted for three different values of the shear angle at the origin, namely $\gamma_{0}=0^{\circ}$ (poloidal field), $\gamma_{0}=10^{\circ}$ and $\gamma_{0}=20^{\circ}$.

selecting this particular form for the Alfvén speed is that, such as shown by Terradas et al. (1999), the vertical behaviour of fast global modes in a coronal potential arcade is completely determined by $\delta$ in such a way that an arcade in which the Alfvén speed increases with height $(\delta>2$ in Eq. (13)) is characterised by vertically evanescent fast-mode solutions, which could be in principle more feasible for observational detection since they are confined within the solar corona. On the other hand, an arcade in which the Alfvén speed decreases with height $(\delta<2$ in Eq. (13)) has solutions whose energy tends to escape toward large heights, making their observational detection difficult. Here we have considered the first case, and the value $\delta=6$ has been used in all our computations. Moreover, this value of $\delta$ ensures that the equilibrium density decreases with height within a wide range of values of the magnetic shear.

\subsection{Linear ideal MHD wave equations}

The equilibrium configuration described above is a particular case of the general two-dimensional force-free equilibrium with invariance along the axis of the structure for which the linear and adiabatic MHD wave equations where derived by Arregui et al. (2001). The physically significant expressions are obtained by projecting all vectors in the directions defined at each point by the unit vectors (see Fig. 2) $\hat{\boldsymbol{e}}_{n}=\nabla A /|\nabla A|$ (normal to magnetic surfaces), $\hat{\boldsymbol{e}}_{\|}=\boldsymbol{B} / \boldsymbol{B}$ (parallel to magnetic field lines) and $\hat{\boldsymbol{e}}_{\perp}=\hat{\boldsymbol{e}}_{\|} \times \hat{\boldsymbol{e}}_{n}$ (tangential to magnetic surfaces and perpendicular to field lines). Since the equilibrium configuration is $y$-invariant, we can Fourier-analyse the perturbed velocity with respect to this coordinate and make it proportional to $\exp \left(i k_{y} y\right)$. Then, the linear and adiabatic MHD wave equations for adiabatic changes of state can be cast as two second-order partial differential equations for the components of the perturbed velocity in the directions normal, $v_{n}$, and perpendicular, $v_{\perp}$, to the

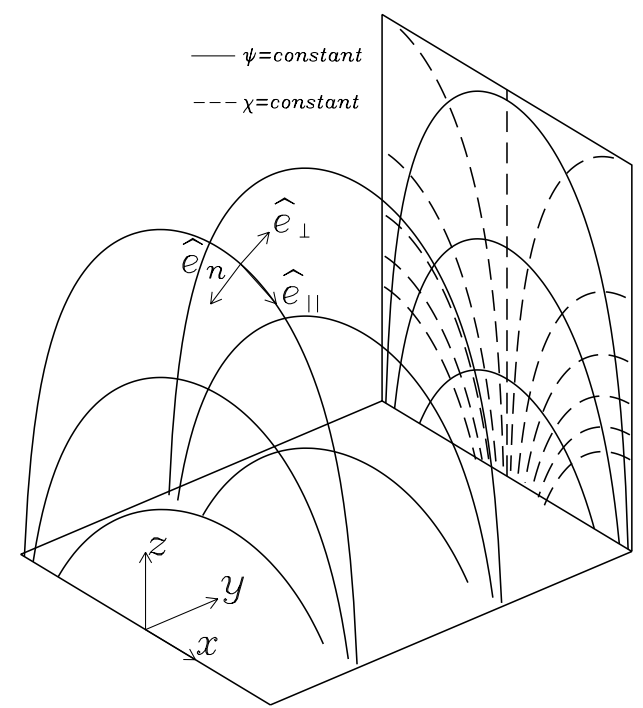

Fig. 2. Three-dimensional view of some magnetic field lines in a sheared potential arcade. The field related vectors $\hat{\boldsymbol{e}}_{n}, \hat{\boldsymbol{e}}_{\perp}$, and $\hat{\boldsymbol{e}}_{\|}$are plotted at a particular point. Also, lines of constant $\psi$ and $\chi$ given by Eqs. (16) and (17) are displayed in the $x z$-plane in which these flux coordinates are used for the numerical computation of eigenmodes.

equilibrium magnetic field,

$\omega^{2} v_{n}=\mathcal{A} v_{n}+\mathcal{B} v_{\perp}$,

$\omega^{2} v_{\perp}=\mathcal{C} v_{n}+\mathcal{D} v_{\perp}$

where $\omega^{2}$ is the squared frequency and $\mathcal{A}, \mathcal{B}, C$ and $\mathcal{D}$ are complicated second order differential operators with derivatives along the equilibrium magnetic field $(\boldsymbol{B} \cdot \nabla)$ and across magnetic surfaces $(\nabla A \cdot \nabla)$ acting on $v_{n}$ and $v_{\perp}$ (see Arregui et al. 2001, for their explicit form). When both $B_{y}=0$ and $k_{y}=0$ these equations are decoupled. Fast modes are then 
characterised by motions in the normal direction $\left(v_{\perp}=0\right.$, $v_{n} \neq 0$ ) whereas Alfvén modes are characterised by motions in the perpendicular direction $\left(v_{n}=0, v_{\perp} \neq 0\right)$. As the plasma- $\beta$ is zero, $v_{\|}=0$ and the slow mode is absent. Whenever longitudinal propagation $\left(k_{y}\right)$ and/or magnetic field shear $\left(B_{y}\right)$ are present, Eqs. (14) and (15) are coupled and, hence, describe coupled fast and Alfvén modes.

\subsection{Numerical method and boundary conditions}

The wave Eqs. (14) and (15) are too complicated to have analytical solutions, hence solutions are obtained by numerical means. The numerical code we use was developed by Oliver et al. (1996) to solve the linear fast and slow mode equations in a two-dimensional equilibrium with no gravity. Finite differences are used to replace the derivatives of $v_{n}$ and $v_{\perp}$, so the problem reduces to solving an algebraic sparse and complex eigenvalue system in which the unknowns are the eigenfrequency and the values of $v_{n}$ and $v_{\perp}$ over a grid of points in the $x z$-plane. By solving this eigenvalue problem with the appropriate boundary conditions, the output consists of the eigenfrequency and the two-dimensional spatial distribution of the perturbed velocity components. The code in its previous version has been successfully used for the study of the properties of linear waves in several poloidal equilibria, i.e. with no longitudinal magnetic field component. For example Smith et al. (1997) used the code for the study of the ducting properties of fast waves in coronal loops and Smith et al. (1998) studied the properties of ULF waves in the Earth magnetosphere. Later, the code was modified by Arregui et al. (2001) in order to include the new terms that arise by the consideration of longitudinal magnetic field component and longitudinal propagation, and a staggered mesh arrangement was included (Arregui et al. 2003) for the proper computation of modes with singular behaviour on magnetic surfaces, such as Alfvén continuum modes.

In a curved magnetic structure, such as the one described in Sect. 2.1, it is very useful to use generalised coordinates to map the part of the $x z$-plane in which the solution is to be computed. These coordinates, for which the names $\psi$ and $\chi$ are selected, can be chosen so as to be flux coordinates linked to the shape of magnetic field lines. From the expression for the flux function, given by Eq. (8), these coordinates are defined here as

$$
\begin{array}{lr}
\psi(x, z)=\cos k x \mathrm{e}^{-k z}, & 0 \leq \psi \leq 1, \\
\chi(x, z)=\frac{\sin k x \mathrm{e}^{-k z}}{\left(1-\psi^{2}\right)^{1 / 2}}, & -1 \leq \chi \leq+1 .
\end{array}
$$

Figure 2 shows $\psi=$ constant and $\chi=$ constant lines for these flux coordinates. With these coordinates, the curved structure of the arcade is transformed into a straight field configuration contained in a box (see Arregui et al. 2004). Flux coordinates are required due to the strongly anisotropic nature of Alfvén modes. Moreover, they allow us to consider an arcade of infinite extension in the vertical direction. On the other hand, Cartesian coordinates can in principle be used for the computation of isotropic fast modes, such as was done by Terradas et al. (1999) in their study of the normal modes of oscillation of a magnetostatic arcade. Here coupled fast and Alfvén modes are to be computed and hence we are forced to use flux coordinates given by Eqs. (16) and (17). Smith et al. (1997) and Arregui et al. (2004) showed that fast modes can also be computed in these coordinates, although in this case the spatial structure of $v_{n}$ is not a separable function of the $\psi$ and $\chi$-coordinates.

Now, the photosphere is modelled by the boundaries $\chi=$ -1 for $x / L<0$ and $\chi=1$ for $x / L>0$. On these boundaries, because of the large photospheric inertia compared with the coronal one, it is usually assumed that the photosphere can be treated as a rigid, perfectly reflecting boundary. Hence, in our numerical computations we consider
$v_{n}=v_{\perp}=0$
for
$\chi= \pm 1$

On the other hand, the line $\psi=1$ corresponds to the point $x=0$ and $z=0$, that is, to the top of a field line touching the photosphere from below at a single point. We therefore consider

$$
v_{n}=v_{\perp}=0 \quad \text { for } \quad \psi=1 .
$$

Finally, the boundary $\psi=0$ represents the field line emerging at the point $x / L=-1$, going up to infinity and then returning back to the photosphere at the point $x / L=1$. Actually, and to avoid numerical problems, this boundary is moved to $\psi=\psi_{0}$, with $\psi_{0}$ a small positive quantity. As a consequence the lateral and top boundaries of the system in the $x z$-plane are transformed into a magnetic surface with maximum height $z_{\max }=-1 / 2 k \ln \left(\psi_{0}^{2}\right)$. For the value $\psi_{0}=10^{-10}$ chosen in all the computations shown in this paper, this results in a maximum height of the arcade $z_{\max } / L=14.66$, which is large enough for the proper computation of vertically evanescent global modes. On this boundary, the following condition is imposed

$$
v_{n}=0 \quad \text { for } \quad \psi=\psi_{0} .
$$

\section{Numerical results}

In this section numerical results are shown for coupled fast and Alfvén modes in our sheared potential configuration. As has already been stated, Eqs. (14) and (15) form a set of two coupled partial differential equations describing fast global modes and Alfvén continuum modes. As soon as $B_{y} \neq 0$ and/or $k_{y} \neq 0$, mode coupling occurs and the resulting oscillatory modes have both $v_{n} \neq 0$ and $v_{\perp} \neq 0$. Therefore, no pure modes exist in such configurations. Our purpose is to start from the case $B_{y}=0$, $k_{y}=0$, in which fast and Alfvén modes are decoupled, and then to study the modification of the two modes when one of these parameters or both is different from zero. Our interest, in this work, lies in the strong coupling between fast and Alfvén modes, so the procedure will be to consider a given fast mode and then to compute numerical solutions for $B_{y} \neq 0$ and/or $k_{y} \neq 0$ near its eigenfrequency. The oscillatory properties of weakly coupled fast and Alfvén modes were studied by Arregui et al. (2003) in an equilibrium with a straight, uniform, inclined magnetic field in order to ascertain the effects of $B_{y}$ and $k_{y}$ on the frequency and spatial structure of fast and Alfvén modes. 


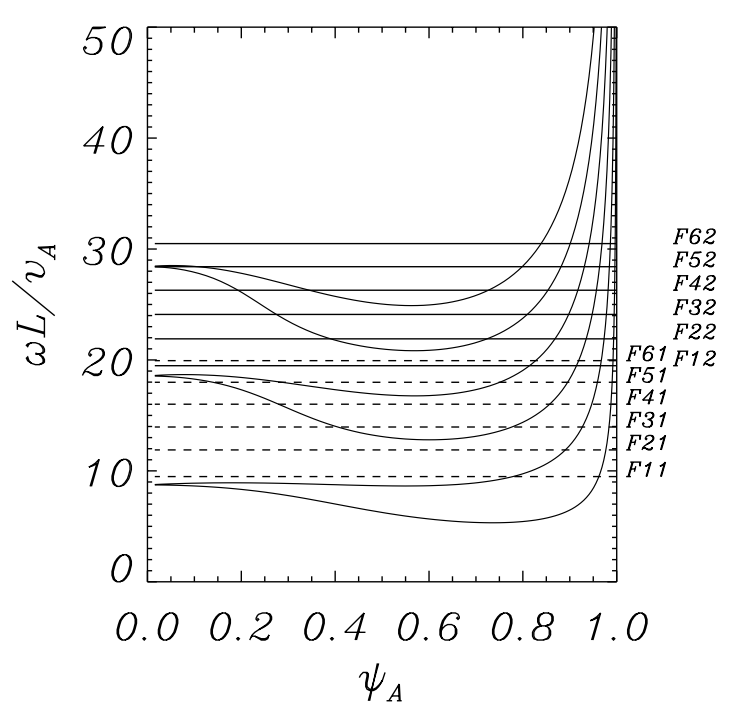

Fig. 3. Fast mode and Alfvén continuum frequencies in a purely poloidal magnetic field configuration $\left(B_{y}=0\right)$ with no longitudinal propagation $\left(k_{y}=0\right)$ (from Arregui et al. 2004). Alfvén mode frequencies are shown as a function of the flux coordinate of the magnetic surface, $\psi_{\mathrm{A}}$, for the first 6 harmonics $\left(n_{\chi}=1,2, \ldots, 6\right.$, where $n_{\chi}$ is the number of extrema of the eigenfunction $v_{\perp}$ in the direction along the equilibrium magnetic field). For a given $\psi_{\mathrm{A}}$, the continuum frequency increases with $n_{\chi}$. Fast modes have been computed using flux coordinates on a mesh of $N_{\chi}=N_{\psi}=61$ points. For these modes, the number of extrema of the eigenfunction $v_{n}$ in the $x$ - and $z$-directions are $n_{x}$ and $n_{z}$. Only fast modes with $n_{z}=1$ and 2 are displayed and their frequencies are over-plotted as horizontal dashed $\left(n_{z}=1\right)$ and solid $\left(n_{z}=2\right)$ lines. The labels F11, F12, .. on the right-hand side of this figure are of the form $\mathrm{F} n_{x} n_{z}$ and so specify the spatial structure of the fast mode eigenfunction.

The spectrum of an unsheared coronal potential arcade with $k_{y}=0$ is shown in Fig. 3. As shown by Oliver et al. (1993) (see also Arregui et al. 2001), fast global modes in a potential arcade $\left(B_{y}=0\right)$ with $k_{y}=0$ are characterised by a discrete spectrum of frequencies that can be classified in terms of the global spatial distribution of the normal velocity component by means of the number of extrema in the $x$ - and $z$-directions, $n_{x}$ and $n_{z}$. Now fast modes are computed in flux coordinates and so the spatial distribution of $v_{n}$ is not a separable function of $\psi$ and $\chi$. Therefore, fast modes cannot be characterised by the number of extrema of their eigenfunctions in the $\psi$ - and $\chi$-directions as these numbers are meaningless. However, these modes have a given parity or symmetry with respect to the plane $\chi=0$ (which is the same as the plane $x=0$ ). On the other hand, Alfvén continuum modes are characterised by the number of extrema in the $\chi$-direction, $n_{\chi}$, on a given magnetic surface. Changing the footpoint position (or the corresponding magnetic flux surface, described by $\psi=\psi_{\mathrm{A}}$ ) produces a continuous change of the Alfvén mode frequency, leading to the continuous spectrum for different values of $n_{\chi}$. Some fast mode frequencies are shown as horizontal lines in this figure. Arregui et al. (2004) pointed out the importance of Fig. 3 claiming that it suffices for a complete qualitative explanation of the spatial structure of coupled fast and Alfvén modes of the system.
When coupling occurs modes appear as pairs with very similar frequency and spatial structure. A simple way to distinguish between fast and Alfvén modes is to consider their compressibility. However, it turns out that under the conditions of strong coupling it is impossible to make a clear distinction between these modes because the spatial structure of both the perturbed quantities (perturbed density and magnetic pressure) and perturbed forces (gradient of the magnetic pressure and magnetic tension) is almost indentical. This fact was shown by Arregui et al. (2003). Figure 13 in that paper shows that the perturbed density for two strongly coupled fast and Alfvén modes is almost identical. A similar situation was studied by Tsiklauri \& Nakariakov (2002) who found that the linear coupling of Alfvén and fast waves leads to the formation of an essentially compressible MHD wave form. In this sense, the normal modes considered here could be regarded as standing analogs of the propagating MHD wave forms discussed by Tsiklauri \& Nakariakov (2002). However, attending to their origin, one of these modes could be termed the more "fast-like" mode and the other one the "Alfvén-like" mode, although as we have already stated there are no pure fast nor pure Alfvén modes. In the following two sections the study of the spatial structure of the perturbed velocity components for the fast-like mode is presented as a function of the presence or absence of $B_{y}$ and $k_{y}$, the parity of the original fast and Alfvén eigenfunctions and the occurrence or absence of resonant and non-resonant couplings. In general there are three cases depending on whether only $B_{y} \neq 0$, only $k_{y} \neq 0$ or both longitudinal propagation and longitudinal magnetic field component are present. The case $B_{y}=0, k_{y} \neq 0$ has already been studied by Arregui et al. (2004) and, hence, it will be not considered here.

\subsection{Potential arcade with sheared magnetic field}

We first consider the effect of the longitudinal magnetic field component or magnetic field shear on the properties of the oscillatory modes and, hence, take $k_{y}=0$ and let $B_{y} \neq 0$. To examine the effect of $B_{y}$ on the spatial distribution of the velocity, we consider the fast mode with $n_{x}=2$ and $n_{z}=1$ and, starting from the case $B_{y}=0$, for which $\gamma_{0}=0^{\circ}$, we steadily increase this parameter and follow the evolution of the fast mode solution for different values of $\gamma_{0}$. As soon as $B_{y}$ is made different from zero, numerical solutions are characterised by real and non-zero $v_{n}$ and $v_{\perp}$. Figures $4 \mathrm{a}$ and $\mathrm{b}$ show the spatial distribution of the perturbed velocity for $\gamma_{0}=10^{\circ}$. As can be appreciated, the normal component of the velocity displays a global spatial distribution in flux coordinates. On the other hand, the perpendicular velocity component shows a different behaviour in different parts of the computational box: a singularity with $n_{\chi}=1$ can be clearly seen near the bottom $(\psi \simeq 1)$ of the arcade, whereas a global behaviour around $\psi \simeq 0.5$ is also present. This global part of the eigenfunction has three extrema in the direction along the magnetic field, so corresponds to a collection of $n_{\chi}=3$ Alfvén continuum solutions. The large velocity values observed towards $\psi=0$ are surely of numerical origin since that boundary corresponds to the magnetic surface that extends up to infinite height. Following the interpretation 


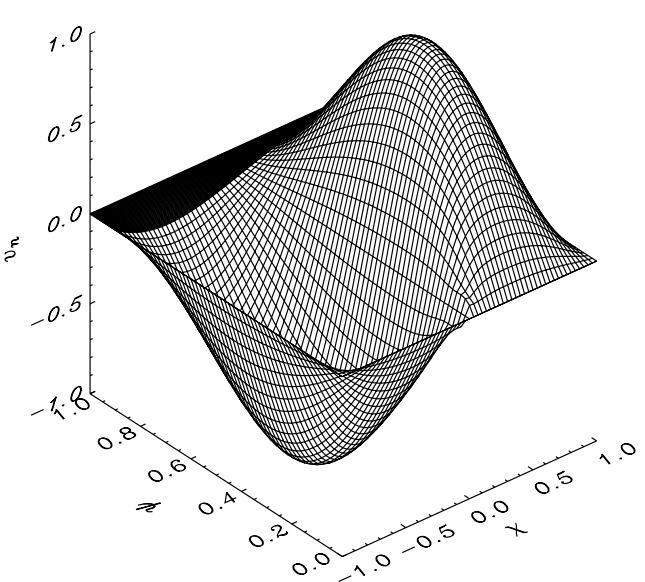

(a)

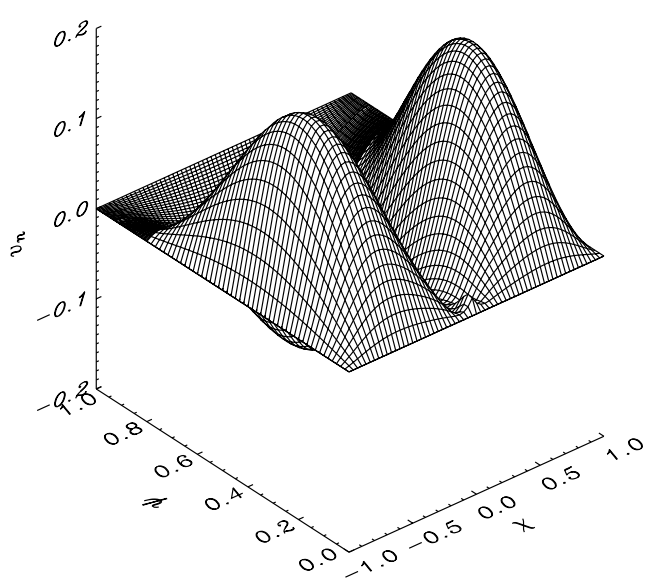

(c)

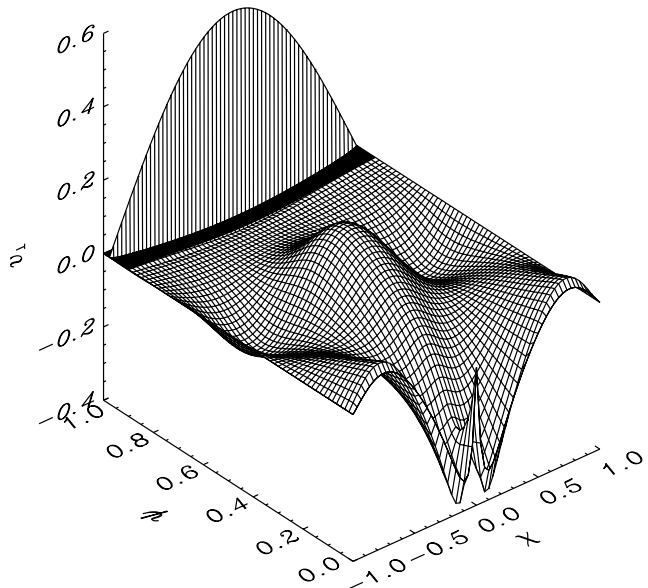

(b)

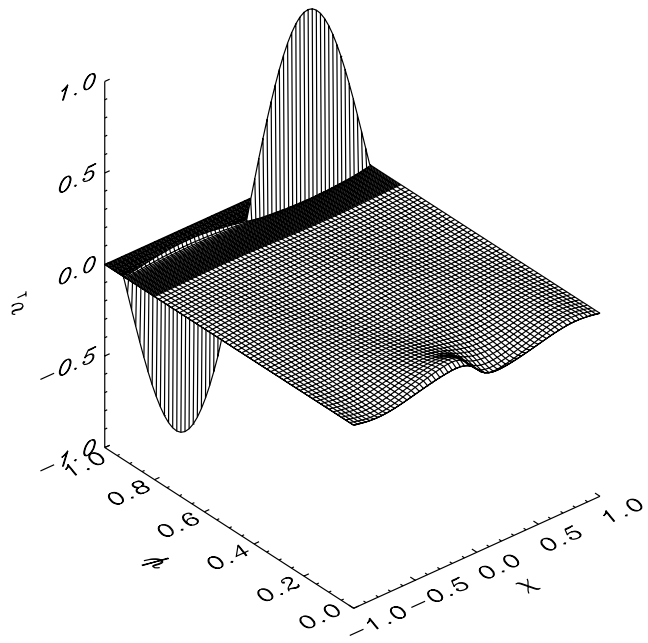

(d)

Fig. 4. a), b) Fast mode-like solution coming from the coupling of the fast mode with $n_{x}=2, n_{z}=1$ and Alfvén continuum modes in a sheared potential coronal arcade (with $\gamma_{0}=10^{\circ}$ ) with no longitudinal propagation and $k_{y}=0$. a) Normal velocity component and b) perpendicular velocity component. The occurrence of two types of coupling can be inferred from the spatial structure of $v_{\perp}$ in $\mathbf{b}$ ): resonant coupling with the Alfvén continuum mode with $n_{\chi}=1$ and matching frequency at $\psi_{\mathrm{A}}=0.975$ leads to a singular behaviour on that magnetic surface, whereas non-resonant coupling with Alfvén modes with $n_{\chi}=3$ and frequency just above the fast mode frequency gives rise to the smooth global structure centred around $\psi_{\mathrm{A}}=0.5$. This numerical solution has been computed on a non-uniform mesh with $N_{\chi}=61$ points and 48 points in the range $\psi=\psi_{0}-0.9$ and 24 points in the range $\psi=0.9-1.0$. c) and d) Fast mode-like solution coming from the coupling of the fast mode with $n_{x}=3, n_{z}=1$ and Alfvén continuum modes in the same configuration with $\gamma_{0}=10^{\circ}$ and $k_{y}=0$. c) Normal and d) perpendicular velocity components. Here only resonant coupling occurs between the fast mode and an Alfvén continuum mode with $n_{\chi}=2$ and matching frequency at $\psi_{\mathrm{A}}=0.88$. This numerical solution has been computed on a non-uniform mesh with $N_{\chi}=61$ points and 48 points in the range $\psi=\psi_{0}-0.8$ and 36 points in the range $\psi=0.8-1.0$.

given by Arregui et al. (2004) in their study of the coupled fast and Alfvén modes in a linear force-free coronal arcade, and attending to the location of the F 21 fast mode frequency in Fig. 3, we can interpret the global behaviour of $v_{\perp}$ in a range of magnetic surfaces as being due to the non-resonant coupling of the fast mode with a group of Alfvén continuum modes with $n_{\chi}=3$. Also, by looking to the intersection of the fast mode frequency with the $n_{\chi}=1$ Alfvén continuum in Fig. 3 , the presence of the singular magnetic surface near the bottom of the arcade can be understood as being due to the resonant coupling of the fast mode to an Alfvén continuum mode with $n_{\chi}=1$ and matching frequency on that magnetic surface. Finally, we want to point out that although the fast mode frequency is also inside the $n_{\chi}=2$ Alfvén continuum, no signature of any singularity with $n_{\chi}=2$ is seen in the perpendicular velocity component of this mode. Hence, such as already pointed out by Arregui et al. (2004), the coupling of fast and Alfvén modes for $B_{y} \neq 0$ seems to involve only modes with opposite parity about $\chi=0$ (which is equivalent to the parity about $x=0$ ).

The previous interpretation of resonant and non-resonant coupling and the derived parity rule for coupling due to magnetic field shear are further confirmed by considering a fast mode with even parity with respect to $\chi=0$, namely the fast mode with $n_{x}=3$ and $n_{z}=1$. Figures $4 \mathrm{c}$ and d display the spatial velocity distribution of the resulting oscillatory mode with $B_{y} \neq 0$. The normal component of the velocity has the 
usual smooth global behaviour and its spatial structure is not a separable function of the flux coordinates $\psi$ and $\chi$. On the other hand, the perpendicular velocity component (see Fig. 4d) shows a distribution with a velocity that is almost zero everywhere except on a given magnetic surface in which a singularity with $n_{\chi}=2$ can be appreciated. Therefore, one can confirm that although the frequency of this fast mode with even symmetry on the direction along field lines cuts the Alfvén continua with $n_{\chi}=1,2$ and 3 (at two points in the last case), the coupling only takes place with a continuum mode with matching frequency and $n_{\chi}=2$. In addition, there is no signature of nonresonant coupling in the perpendicular velocity component and this absence of global behaviour on a range of magnetic surfaces is interpreted on the basis of the large distance in Fig. 3 between the fast mode frequency and the first continuum above it with $n_{\chi}$ even, i.e. the $n_{\chi}=4$ continuum. On the basis of the above results one can also confirm the parity rule for coupling due to the presence of magnetic field shear.

\subsection{Potential arcade with sheared magnetic field and longitudinal propagation}

After having studied the properties of the normal modes of oscillation in a sheared potential coronal arcade, we next consider the combined effects of magnetic field shear and longitudinal propagation. As has already been mentioned, the properties of coupled fast and Alfvén modes in a potential arcade in the case in which only longitudinal propagation is present $\left(B_{y}=0\right.$, $k_{y} \neq 0$ ) were studied by Arregui et al. (2004). These authors showed that longitudinal propagation of perturbations only allows for the coupling between fast and Alfvén modes with the same parity with respect to $\chi=0$. Arregui et al. (2004) then considered $B_{y} \neq 0, k_{y} \neq 0$ and observed that the presence of both effects gives rise to the coupling of any kind of mode regardless the parity of their eigenfunctions.

To check whether the previous rule also applies to the present potential equilibrium, the simplest procedure, is to consider one of the solutions displayed in the previous section and, then, to steadily increase the value of the longitudinal wave number, $k_{y}$. To this end, we consider the mode coming from the coupling of the fast mode F21 with one Alfvén continuum mode with $n_{\chi}=1$ and a collection of Alfvén continuum modes with $n_{\chi}=3$, whose spatial structure is depicted in Figs. 4a and $b$. Then, the same mode is computed for increasing values of the parameter $k_{y} L$. Figures $5 \mathrm{a}$ and $\mathrm{b}$ show the spatial distribution of the perturbed velocity components for the resulting oscillatory mode. Now, both $v_{n}$ and $v_{\perp}$ are complex quantities, so their modulus is plotted. Figure 5 a shows that the normal component of the velocity retains its global spatial distribution and no significant changes are observed with respect to Fig. 4a. The only remarkable feature is the presence of a small jump on a magnetic surface with $\psi_{\mathrm{A}} \simeq 0.88$, a common feature of coupled fast and Alfvén modes (Arregui et al. 2004), that is here more evident than in Fig. 4a. On the other hand, the perpendicular velocity component (see Fig. 5b) displays a new feature when compared to the spatial distribution in Fig. 4b. A singularity with $n_{\chi}=2$ is also present together with the singularity with $n_{\chi}=1$ and the global behaviour with $n_{\chi}=3$ that were already present in Fig. 4b. We interpret this result as being due to the resonant coupling of the F21 fast mode with an Alfvén continuum mode with $n_{\chi}=2$. Such as seen in the previous section, this coupling is forbidden by the coupling rule for $B_{y} \neq 0$ and $k_{y}=0$, but this result is completely in accordance with the results of Arregui et al. (2004) in the sense that the new feature is due to the coupling rule for longitudinal propagation derived by these authors. Therefore, the presence of both magnetic field shear and longitudinal propagation $\left(B_{y} \neq 0, k_{y} \neq 0\right)$ allows for any coupling between fast and Alfvén modes regardless the parity or symmetry of their eigenfunctions with respect to $\chi=0$.

This result is further confirmed by considering a fast mode with different parity with respect to $\chi=0$. For the sake of simplicity, we consider the mode for which the spatial structure is shown in Figs. 4c and d. This mode has been computed computed for non-zero values of the longitudinal propagation and the spatial distribution of the velocity for the resulting solution is shown in Figs. 5c and d. Here, a global, smooth spatial structure can be seen in Fig. $5 \mathrm{c}$ for $v_{n}$, whereas the fingerprints of the different couplings that take place with Alfvén continuum modes with different parities can be appreciated in Fig. 5d.

Nevertheless, an important difference can be seen when comparing the normal velocity component for $B_{y} \neq 0, k_{y} \neq 0$ (Fig. 5c) with the one shown for $B_{y} \neq 0, k_{y}=0$ (Fig. 4c). Namely, the spatial distribution of the velocity in the former figure takes significantly large values at large heights in the arcade (near $\psi=0$ ). This energy "leakage" from low to large heights when the equilibrium ceases to be potential was already pointed out by Terradas et al. (1999) when considering the modes of oscillation of a magnetostatic arcade, and more recently by Arregui et al. (2004) in their study of the modes of oscillation of a linear force-free arcade. These authors pointed out that for sufficiently large values of the magnetic field shear and longitudinal wavenumber the mode is no longer evanescent. This property is here well recovered despite the equilibrium configuration considered here is potential, and therefore this property cannot be attributed to the non-potentiality of the equilibrium, but to the shear of magnetic field lines and mode coupling.

Another example of the loss of confinement of coupled fast and Alfvén modes when both magnetic field shear and longitudinal propagation are present is shown in Fig. 6. Figure 6a displays the normal component of the velocity for the mode coming from the coupling of the fast mode with $n_{x}=6$ and $n_{z}=1$ to several Alfvén continuum modes. This figure also shows large velocity values at large heights in the arcade, which is better seen by transforming the spatial distribution of the velocity to Cartesian coordinates (Fig. 6b). Then, the fact that the mode is no longer evanescent is clearly seen. Moreover, we want to remark that, as demonstrated by the spatial distribution of the velocity shown in Figs. 5c and 6a, both even and odd modes suffer from this loss of confinement and, hence, this seems to occur regardless the symmetry of the mode.

Finally, concerning the oscillatory frequency, our results indicate that the frequency of coupled fast-Alfvén modes is real only if magnetic shear or longitudinal propagation are present. 


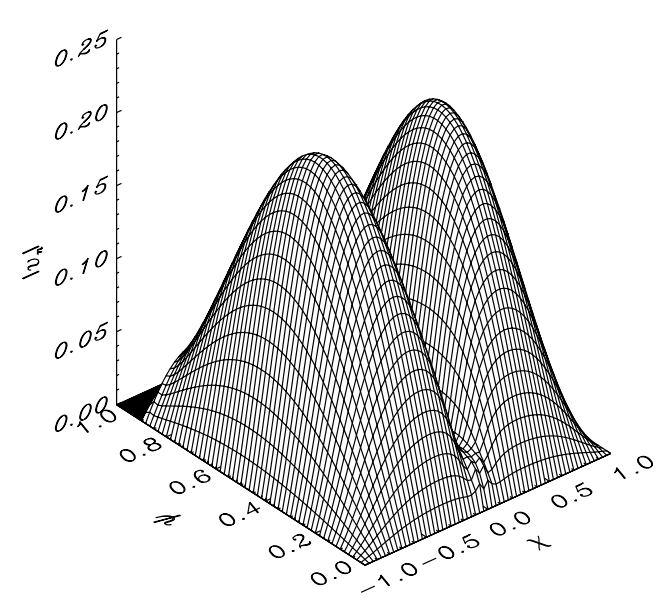

(a)

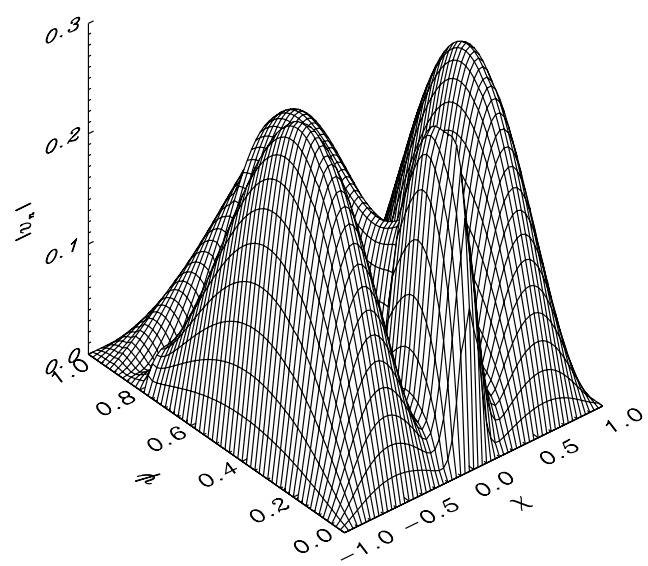

(c)

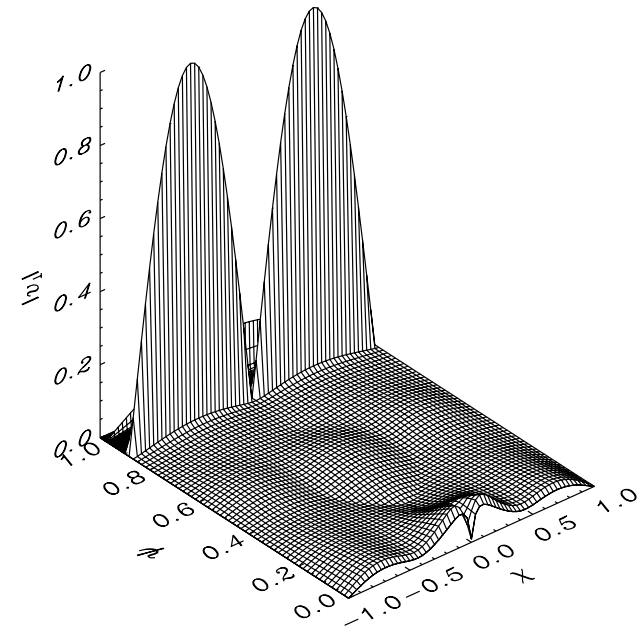

(b)

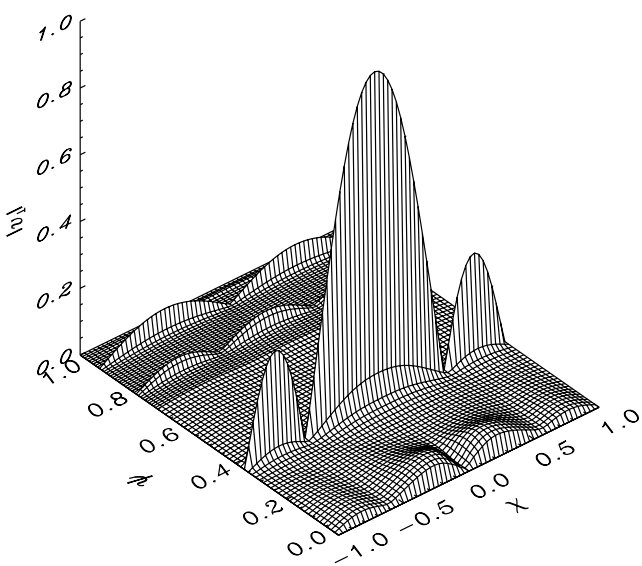

(d)

Fig. 5. a) Normal and b) perpendicular velocity components for the fast mode-like solution coming from the coupling of the fast mode with $n_{x}=2, n_{z}=1$ and three Alfvén continuum modes in a sheared potential coronal arcade $\left(\gamma_{0}=10^{\circ}\right)$ with longitudinal propagation $\left(k_{y} L=1.0\right)$. Resonant coupling takes place with Alfvén continuum modes with $n_{\chi}=1$ and $n_{\chi}=2$ and matching frequency at $\psi_{\mathrm{A}}=0.975$ and $\psi_{\mathrm{A}}=0.88$, respectively, and ordinary coupling takes place with Alfvén continuum modes with $n_{\chi}=3$. This numerical solution has been computed on a non-uniform mesh with $N_{\chi}=61$ points and 48 points in the range $\psi=\psi_{0}-0.8$ and 24 points in the range $\psi=0.8-1.0$. c) and d) Normal and perpendicular velocity components for the fast mode-like solution coming from the coupling of the fast mode with $n_{x}=3, n_{z}=1$ in the same configuration. Resonant coupling with Alfvén continuum modes with $n_{\chi}=1, n_{\chi}=2$ and $n_{\chi}=3$ can be appreciated. This numerical solution has been computed on a uniform mesh with $N_{\chi}=61$ and $N_{\psi}=61$ points.

However, the frequency becomes complex when both magnetic field shear and longitudinal propagation are non-zero. The computed value of the imaginary part of the frequency, $\omega_{\mathrm{I}}$, strongly depends on the computational mesh, so a fixed value cannot be given unless dissipation is included in our numerical computations (see also Arregui et al. 2003, 2004).

\section{Conclusions}

In this paper, the magnetohydrodynamic normal modes of oscillation of a sheared potential coronal arcade have been studied in the cold plasma approximation. To this end, an equilibrium with a constant longitudinal magnetic field has been considered. This leads to a configuration in which magnetic field lines are sheared in such a way that field lines belonging to different magnetic surfaces have a different shear angle.
In the particular configuration used throughout this work, the shear increases from a particular value, $\gamma_{0}$, at the photospheric level up to $90^{\circ}$ for large heights. Therefore, substantial differences could be expected when comparing the normal modes of oscillation of this equilibrium with the ones found for the constant- $\alpha$ linear force-free magnetic equilibrium considered by Arregui et al. (2004), in which magnetic field lines of all magnetic surfaces have the same shear angle. Regarding the plasma variables in the unperturbed state, the equilibrium density and Alfvén speed are only functions of the vertical coordinate. As the plasma- $\beta$ is zero, one of these variables can be freely imposed and, here, the Alfvén speed is assumed to have an exponentially increasing profile in the vertical direction. This selection ensures the confinement of the perturbed velocity of uncoupled global fast modes to low heights in the solar corona. 


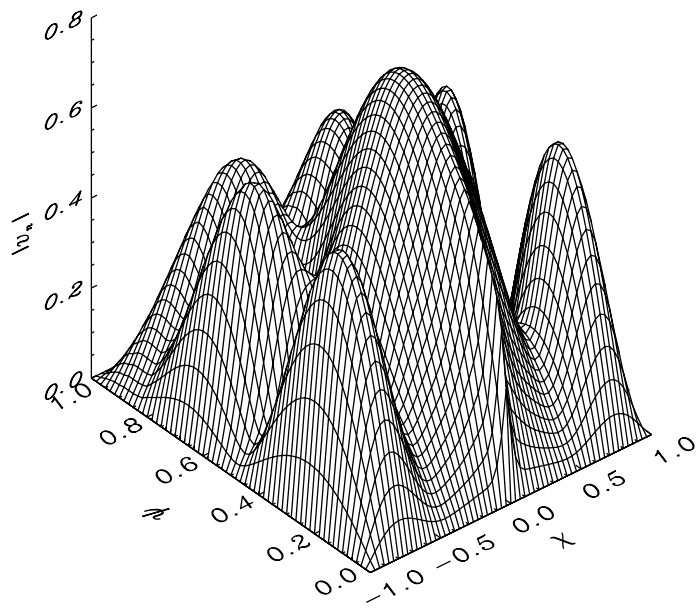

(a)

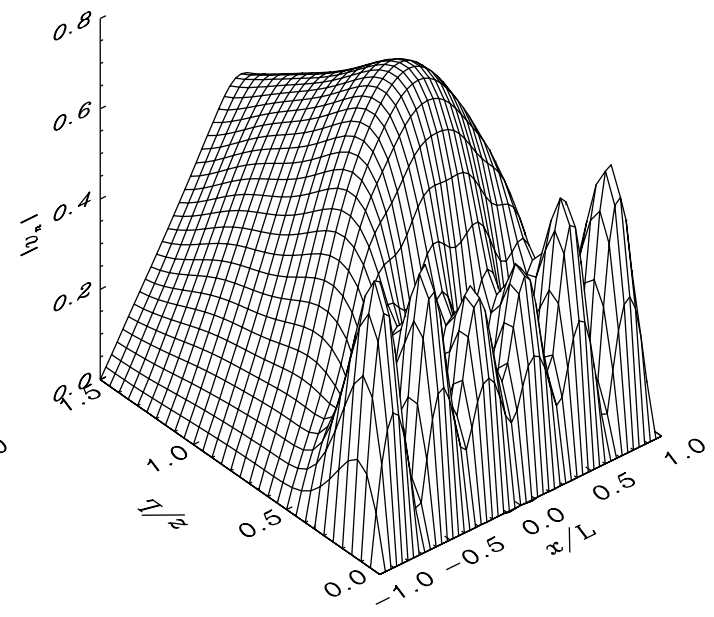

(b)

Fig. 6. Normal velocity component for the coupled solution coming from the fast mode with $n_{x}=6$ and $n_{z}=1$ in a sheared potential arcade $\left(\gamma_{0}=10^{\circ}\right)$ with longitudinal propagation $\left(k_{y} L=0.7\right)$. a) Spatial distribution of the velocity represented in flux coordinates. b) The same spatial distribution transformed to Cartesian coordinates by means of the inverse transformation of Eqs. (16) and (17). Large amplitudes are found at high altitudes and so the mode is no longer vertically evanescent.

The purpose of this study has been to extend the work of Oliver et al. (1993), in which the oscillatory properties of decoupled fast and Alfvén modes were studied in a potential coronal arcade, to the cases in which the equilibrium longitudinal magnetic field component and longitudinal propagation of perturbations are included. As shown by Arregui et al. (2001), these two effects produce the coupling of global fast modes, characterised by a discrete set of frequencies, and singular Alfvén modes, with a continuous range of frequencies, in such a way that no pure fast or Alfvén waves exist, but modes with mixed properties arise. This is important as it constitutes an additional constraint to the diagnostic tool mentioned by Oliver et al. (1993) by which uncoupled fast and Alfvén modes could in principle be detected, attending to the polarisation of the motions at or above the limb. The resulting oscillatory modes, which are neither pure fast nor pure Alfvén modes, display a mixture of properties in such a way that the spatial distribution of the perturbed velocity has a global spatial behaviour together with a singular, localised behaviour on given magnetic surfaces. As shown by Arregui et al. (2003), the coupling between fast and Alfvén modes in a straight uniform magnetic field with inclined magnetic field and longitudinal propagation is governed by some parity rules. Then, similar parity rules were also found by Arregui et al. (2004), who showed that longitudinal propagation produces the coupling of fast and Alfvén modes with the same parity with respect to $\chi=0$. In our sheared potential configuration the parity rule due to a non-zero longitudinal magnetic field component, that states that only modes with opposite parity interact, has been confirmed. Similarly, the fact that any coupling can take place when both $B_{y} \neq 0$ and $k_{y} \neq 0$ has also been established.

Despite the significant differences between the linear forcefree magnetic configuration investigated by Arregui et al. (2004) Arregui et al. (2004) and the sheared potential arcade equilibrium for which the normal modes have been studied here, the results obtained in this paper concerning the coupling rules and the signatures of mode coupling, i.e. the presence or absence of singular contribution(s) from the interaction with Alfvén mode(s) depending on the nature of such a coupling, are the same. This is important since it tells us that both the parity rules as well as the conditions for which resonant or non-resonant coupling occur seem not to be particular properties for these two equilibria, but could be considered as more general features for linear and adiabatic standing waves in twodimensional sheared equilibria with longitudinal propagation in the cold plasma limit.

An important result of this paper concerns the loss of confinement of coupled modes for sufficiently large values of the shear angle and the longitudinal wavenumber. As we have already mentioned, the choice of an exponentially increasing Alfvén speed profile ensures the vertically evanescent behaviour of global modes in a potential arcade when both $B_{y}$ and $k_{y}$ are zero. The fact that this feature does not hold when both effects are present cannot be attributed to a property of the equilibrium configuration, but must be considered a consequence of mode coupling. In addition, an inspection of Figs. 5c and $6 a$ reveals that this loss of confinement, which can be of importance for the detection of this kind of waves in the solar corona, occurs no matter the symmetry or parity of the mode with respect to the direction of the equilibrium magnetic field. Finally, the results obtained in this paper can open the way for further studies of MHD waves in coronal structures. For instance, a similar situation to the "harmonica" event observed by TRACE on April 15, 2001 and mentioned in the introduction can be simulated with the code used in this paper by considering, for example, the oscillations of a coronal arcade with the bright TRACE loops modelled as a density enhancement with longitudinal propagation of waves $\left(k_{y} \neq 0\right)$ along the tunnel of the arcade. Also, these results open the way for the study of the normal modes of oscillation of prominences embedded in a sheared coronal arcade, given the substantial shear of the magnetic field in prominences. 
Acknowledgements. The authors wish to acknowledge the financial support received from the Spanish Ministry of Science and Technology (MCyT) under the project AYA2003-00123.

\section{References}

Arregui, I., Oliver, R., \& Ballester, J. L. 2001, A\&A, 369, 1122 Arregui, I., Oliver, R., \& Ballester, J. L. 2003, A\&A, 402, 1129 Arregui, I., Oliver, R., \& Ballester, J. L. 2004, ApJ, 602, 1020

Aschwanden, M. J. 1987, Sol. Phys., 111, 113

Aschwanden, M. J., Fletcher, L., Schrijver, C. J., \& Alexander, D. 1999, ApJ, 520, 880

Berghmans, D., \& Clette, F. 1999, Sol. Phys., 186, 207

De Moortel, I., Ireland, J., \& Walsh, R. W. 2000, A\&A, 355, L23

De Moortel, I., Hood, A. W., \& Ireland, J. 2002a, A\&A, 381, 311

De Moortel, I., Hood, A. W., Ireland, J., \& Walsh, R. W. 2002b, Sol. Phys., 209, 89

De Moortel, I., Ireland, J., Walsh, R. W., \& Hood, A. W. 2002c, Sol. Phys., 209, 61

DeForest, C. E., \& Gurman, J. B. 1998, ApJ, 501, L217

Egan, T. F., \& Schneeberger, T. J. 1979, Sol. Phys., 64, 223

Goossens, M., Poedts, S., \& Hermans, D. 1985, Sol. Phys., 102, 51

Hansen, P. J., \& Harrold, B. G. 1994, J. Geophys. Res., 99, 2429

Mond, M., Hameiri, E., \& Hu, P. N. 1990, J. Geophys. Res., 95, 89

Nakariakov, V. M., \& Ofman, L. 2001, A\&A, 372, L53
Nakariakov, V. M., Ofman, L., DeLuca, E. E., Roberts, B., \& Davila, J. M. 1999, Science, 285, 862

Ofman, L., Romoli, M., Poletto, G., Noci, G., \& Kohl, J. L. 2000, ApJ, 529,592

Oliver, R., Ballester, J. L., Hood, A. W., \& Priest, E. R. 1993, A\&A, 273, 647

Oliver, R., Hood, A. W., \& Priest, E. R. 1996, ApJ, 461, 424

Poedts, S., \& Goossens, M. 1987, Sol. Phys., 109, 265

Poedts, S., \& Goossens, M. 1991, Sol. Phys., 133, 281

Robbrecht, E., Verwichte, E., Berghmans, D., et al. 2001, A\&A, 370, 591

Roberts, B., Edwin, P. M., \& Benz, A. O. 1984, ApJ, 279, 857

Rosenberg, H. 1970, A\&A, 9, 159

Ruderman, M. S., Goossens, M., Ballester, J. L., \& Oliver, R. 1997, A\&A, 328, 361

Smith, J. M., Roberts, B., \& Oliver, R. 1997, A\&A, 317, 752

Smith, J. M., Wright, A. N., \& Rickard, G. J. 1998, J. Geophys. Res., 103,20515

Terradas, J. 2002, Ph.D. Thesis, Universitat de les Illes Balears

Terradas, J., Oliver, R., \& Ballester, J. L. 1999, ApJ, 517, 488

Tirry, W. J., \& Poedts, S. 1998, A\&A, 329, 754

Tsiklauri, D., \& Nakariakov, V. M. 2002, A\&A, 393, 321

Wang, T. J., Solanki, S. K., Innes, D. E., Curdt, W., \& Marsch, E. 2003, A\&A, 402, L17

Zaqarashvili, T. V. 2003, A\&A, 399, L15 